\title{
On the central configurations in the spatial 5-body problem with four equal masses
}

\section{Martha Alvarez-Ramírez, Montserrat Corbera \& Jaume Llibre}

Celestial Mechanics and Dynamical Astronomy

An International Journal of Space Dynamics

ISSN 0923-2958

Celest Mech Dyn Astr

DOI 10.1007/s10569-015-9670-z

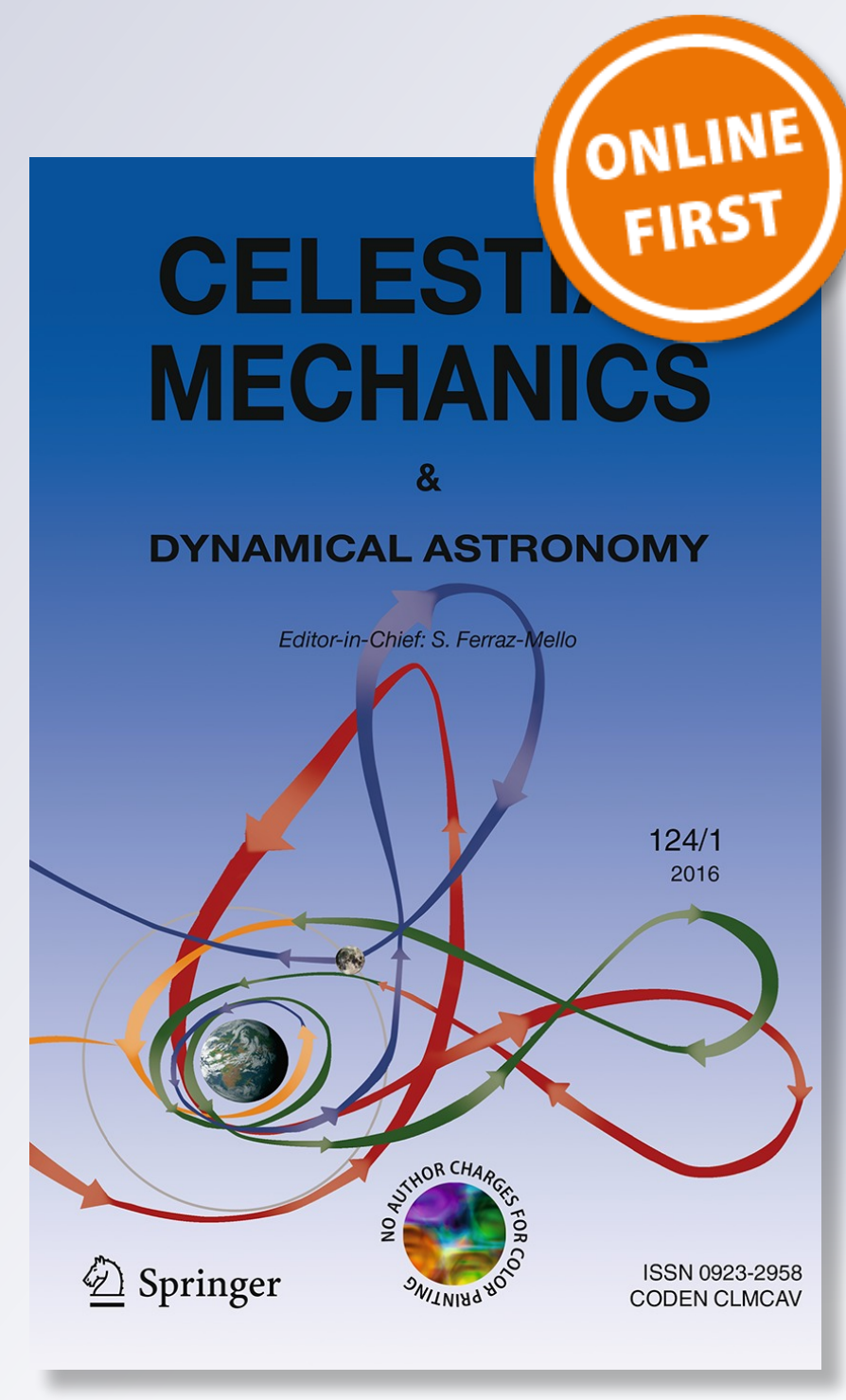

黛 Springer 
Your article is protected by copyright and all rights are held exclusively by Springer Science +Business Media Dordrecht. This e-offprint is for personal use only and shall not be selfarchived in electronic repositories. If you wish to self-archive your article, please use the accepted manuscript version for posting on your own website. You may further deposit the accepted manuscript version in any repository, provided it is only made publicly available 12 months after official publication or later and provided acknowledgement is given to the original source of publication and a link is inserted to the published article on Springer's website. The link must be accompanied by the following text: "The final publication is available at link.springer.com". 


\title{
On the central configurations in the spatial 5-body problem with four equal masses
}

\author{
Martha Alvarez-Ramírez ${ }^{1}$. Montserrat Corbera ${ }^{2}$. \\ Jaume Llibre ${ }^{3}$
}

Received: 1 August 2015 / Revised: 7 December 2015 / Accepted: 30 December 2015

(C) Springer Science+Business Media Dordrecht 2016

\begin{abstract}
We analyze the families of central configurations of the spatial 5-body problem with four masses equal to 1 when the fifth mass $m$ varies from 0 to $+\infty$. In particular we continue numerically, taking $m$ as a parameter, the central configurations (which all are symmetric) of the restricted spatial $(4+1)$-body problem with four equal masses and $m=0$ to the spatial 5-body problem with equal masses (i.e. $m=1$ ), and viceversa we continue the symmetric central configurations of the spatial 5-body problem with five equal masses to the restricted $(4+1)$-body problem with four equal masses. Additionally we continue numerically the symmetric central configurations of the spatial 5-body problem with four equal masses starting with $m=1$ and ending in $m=+\infty$, improving the results of AlvarezRamírez et al. (Discrete Contin Dyn Syst Ser S 1: 505-518, 2008). We find four bifurcation values of $m$ where the number of central configuration changes. We note that the central configurations of all continued families varying $m$ from 0 to $+\infty$ are symmetric.
\end{abstract}

Keywords Spatial central configurations $\cdot 5$-Body problem $\cdot$ Bifurcations

Montserrat Corbera

montserrat.corbera@uvic.cat

Martha Alvarez-Ramírez

mar@xanum.uam.mx

Jaume Llibre

jllibre@mat.uab.cat

1 Departamento de Matemáticas, UAM-Iztapalapa, San Rafael Atlixco 186, Col. Vicentina, Iztapalapa, 09340 Mexico, D.F., Mexico

2 Departament de Tecnologies Digitals i de la Informació, U Science Tech, Universitat de Vic-Universitat Cental de Catalunya (UVic-UCC), C/. Laura, 13, 08500 Vic, Barcelona, Spain

3 Departament de Matemàtiques, Edifici C, Facultat de Ciències, Universitat Autònoma de Barcelona, 08193 Bellaterra, Barcelona, Spain 


\section{Introduction}

A configuration of $n$ bodies is central if the force due to acceleration on each body is proportional to the position vector of the body with respect to the center of mass. Central configurations are very important in the study of the $n$-body problem; for instance, they allow to compute all the homographic solutions, every motion starting and ending in a total collision is asymptotic to a central configuration, and every parabolic motion of the $n$ bodies is asymptotic to a central configuration (Saari and Hulkower 1981; Chenciner 1998). For the 3-body problem the set of central configurations is completely known, but the problem of finding the central configurations when $n>3$ is far to be completely solved. We only know the entire set of central configurations in some particular cases where some of the masses are equal or the configurations satisfy some geometrical properties.

The objective of this paper is to study the central configurations of the spatial 5-body problem with four equal masses when the non equal mass varies from 0 to infinity. More precisely, we will continue numerically all the symmetric central configurations of the spatial 5-body problem with the five masses equal to 1 to the restricted spatial $(4+1)$-body problem with four masses equal to 1 and a fifth infinitesimal mass, and vice versa; that is, we vary the non equal mass from 1 to 0 , and viceversa. This study completes the one presented in Alvarez-Ramírez et al. (2008) where the authors continue numerically the symmetric central configurations from the spatial 5-body problem with the five masses equal to 1 to the restricted spatial $(1+4)$-body problem with four infinitesimal masses equal to $m=0$ and a fifth mass equal to 1 . Note that the study in Alvarez-Ramírez et al. (2008) is equivalent to study the symmetric central configurations of the 5-body problem with four masses equal to 1 varying the fifth mass $1 / m$ from 1 to infinity. This explains why the work of this paper completes the study of Alvarez-Ramírez et al. (2008). Thus the work done in Alvarez-Ramírez et al. (2008) and the one in the present paper provide a skeleton of the families of symmetric central configurations of the spatial 5-body problem with four equal masses and their bifurcations. The results that we have obtained are represented in Fig. 1. Each one of the families of classes of central configurations which appear in Fig. 1 are described later on.

This paper is structured as follows, in Sect. 2 we provide the equations for the central configurations of the spatial 5-body problem. In Sect. 3 we give a brief summary of the known results on symmetric central configurations for the spatial 5-body problem with five equal masses, we provide all the symmetric classes of central configurations of this problem and the positions of a representative of each class. In Sect. 4 we give all the classes of central configurations of the spatial restricted $(4+1)$-body problem with four equal masses and one infinitesimal, and we also give a representative of each class. In Sect. 5 we continue numerically the known families of central configurations from the spatial restricted $(4+1)$ body problem with 4 equal masses to the spatial 5-body problem with equal masses, and vice versa, taking one of the masses $m$ as a parameter. The results that we obtain are summarized in Sect. 6. In Sect. 7 we revisit and improve the work in Alvarez-Ramírez et al. (2008).

\section{Equations of central configurations in the 5-body problem}

The spatial 5-body problem is given by

$$
m_{i} \ddot{\mathbf{q}}_{i}=-\sum_{\substack{j=1 \\ j \neq i}}^{5} G m_{i} m_{j} \frac{\mathbf{q}_{i}-\mathbf{q}_{j}}{r_{i j}^{3}}, \quad i=1, \ldots, 5,
$$




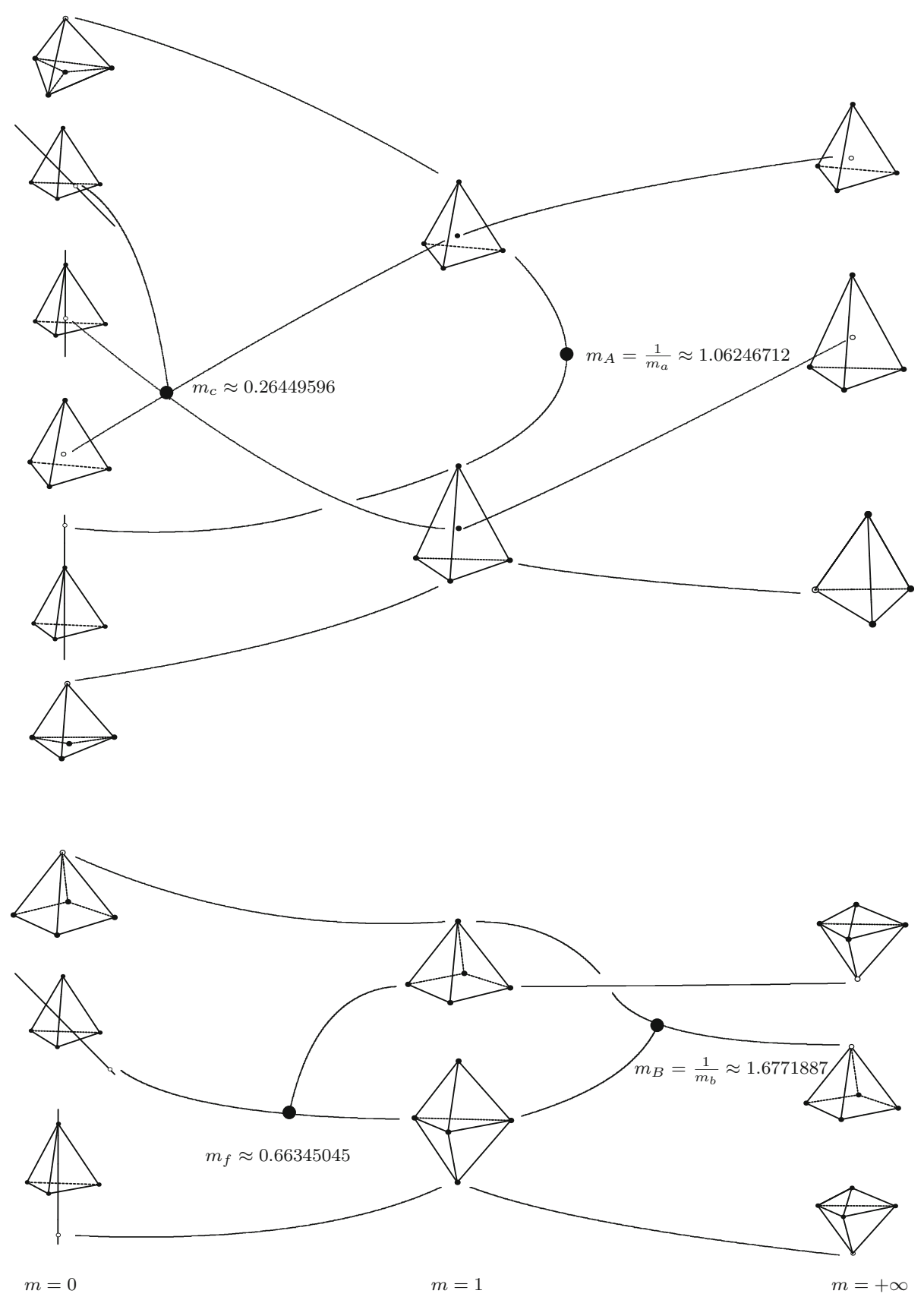

Fig. 1 Connections between the families of classes of central configurations of the 5-body problem with four equal masses when the non-equal mass goes from 0 to $+\infty$. The four equal masses are represented by a small black ball whereas the non-equal mass is represented by a circle 
where $\mathbf{q}_{i} \in \mathbb{R}^{3}$ is the position vector of the body with mass $m_{i}$ in an inertial coordinate system, $r_{i j}=\left|\mathbf{q}_{i}-\mathbf{q}_{j}\right|$ is the distance between the masses $m_{i}$ and $m_{j}$, and $G$ is the gravitational constant which can be taken equal to one by scaling the time.

In these coordinates the configuration space of the spatial 5-body problem is

$$
\mathcal{P}=\left\{\left(\mathbf{q}_{1}, \ldots, \mathbf{q}_{5}\right) \in \mathbb{R}^{3 n}: \mathbf{q}_{i} \neq \mathbf{q}_{j}, \text { for } i \neq j\right\} .
$$

It is known that given $m_{1}, \ldots, m_{5}$ the configuration $\left(\mathbf{q}_{1}, \ldots, \mathbf{q}_{5}\right) \in \mathcal{P}$ is central if the acceleration vector of each body is a common scalar multiple of its position vector (with respect to the center of mass). That is, if there exists a positive constant $\lambda$ such that

$$
\ddot{\mathbf{q}}_{i}=-\lambda\left(\mathbf{q}_{i}-\mathbf{q}_{m}\right), \quad i=1, \ldots, 5,
$$

where

$$
\mathbf{q}_{m}=\frac{\sum_{i=1}^{5} m_{i} \mathbf{q}_{i}}{\sum_{i=1}^{5} m_{i}}
$$

is the position vector of the center of mass of the system. In other words, the given configuration $\left(\mathbf{q}_{1}, \ldots, \mathbf{q}_{5}\right) \in \mathcal{P}$ of the 5-body problem with positive masses $m_{1}, \ldots, m_{5}$ is central if there exists $\lambda$ such that $\left(\lambda, \mathbf{q}_{1}, \ldots, \mathbf{q}_{5}\right)$ is a solution of the system

$$
\lambda\left(\mathbf{q}_{i}-\mathbf{q}_{m}\right)=\sum_{\substack{j=1 \\ j \neq i}}^{5} m_{j} \frac{\mathbf{q}_{i}-\mathbf{q}_{j}}{r_{i j}^{3}}, \quad i=1, \ldots, 5 .
$$

We say that two central configurations belong to the same class if they are invariant under rotations, scaling and permutations of the particles with equal masses.

We assume that the center of mass $\mathbf{q}_{m}$ of the system is fixed at the origin of coordinates. Then system (1) can be written as

$$
\mathbf{f}_{i}=0, \quad i=1, \ldots, 5,
$$

where

$$
\mathbf{f}_{i}=\sum_{\substack{j=1 \\ j \neq i}}^{5} m_{j} \frac{\mathbf{q}_{i}-\mathbf{q}_{j}}{r_{i j}^{3}}-\lambda \mathbf{q}_{i}
$$

Since the center of mass is at the origin the following linear combination of the $\mathbf{f}_{i}$ 's

$$
m_{1} \mathbf{f}_{1}+m_{2} \mathbf{f}_{2}+m_{3} \mathbf{f}_{3}+m_{4} \mathbf{f}_{4}+m_{5} \mathbf{f}_{5}=0,
$$

is satisfied. So, if the mass $m_{i} \neq 0$ then the vectorial function $\mathbf{f}_{i}$ is a linear combination of the other ones, and we can eliminate the equation $\mathbf{f}_{i}=0$ from system (2).

Without loss of generality we can assume that the mass $m_{1}$ is fixed at $\mathbf{q}_{1}=(0,0,1)$. We also can fix $x_{3}=0$, this avoids the rotation of the configuration except when the mass $m_{3}$ is located on the $z$-axis. In this last case, to avoid rotations we should fix a variable different from $x_{3}$. Moreover if $m_{i} \neq 0$ we can isolate $\left(x_{i}, y_{i}, z_{i}\right)$ from the equation $\mathbf{q}_{m}=0$. Thus, assuming that $m_{2} \neq 0$, we can fix the variables $x_{2}, y_{2}$ and $z_{2}$ as follows

$$
\begin{aligned}
& x_{2}=-\frac{m_{4} x_{4}+m_{5} x_{5}}{m_{2}}, \quad y_{2}=-\frac{m_{3} y_{3}+m_{4} y_{4}+m_{5} y_{5}}{m_{2}}, \\
& z_{2}=-\frac{m_{1}+m_{3} z_{3}+m_{4} z_{4}+m_{5} z_{5}}{m_{2}} .
\end{aligned}
$$


On the other hand, from the third component of the vectorial equation $\mathbf{f}_{1}=0$ we get

$$
\lambda=-\sum_{i=2}^{5} \frac{m_{i}\left(z_{i}-1\right)}{\left(x_{i}^{2}+y_{i}^{2}+\left(z_{i}-1\right)^{2}\right)^{3 / 2}}
$$

Therefore system (2) can be reduced to a set of eleven equations, denoted by $e_{i}$ for $i=$ $1, \ldots 11$, and eight unknowns, namely, the position variables $y_{3}, z_{3}, x_{4}, y_{4}, z_{4}, x_{5}, y_{5}, z_{5}$. Clearly these eleven equations are not all independent.

We note that if $m_{2}=0$, then we can proceed in the same way by taking a mass $m_{i} \neq 0$ instead of $m_{2}$ and we would arrive to a similar set of equations but with different unknowns.

In short the problem of finding the central configurations of the 5-body problem is reduced to find the solutions of a system of eleven equations and eight unknowns.

\section{Spatial central configurations in the 5-body problem with equal masses}

Faugère and Kotsireas (1999), and Kotsireas and Lazard (2002) by using linear algebra and Gröbner bases studied the central configurations of the spatial 5-body problem with equal masses. In Faugère and Kotsireas (1999) the authors showed that a convex central configuration of this problem has always a plane of symmetry, see also the Habilitation Thesis of Albouy, section 3.4, first theorem, page 92 (Albouy 1997). In Kotsireas and Lazard (2002) the authors classified the symmetric spatial central configurations with axial symmetry in the 5-body problem with equal masses. In particular, they found four classes of central configurations having an axis of symmetry, two convex and two concave (see Fig. 2), and they conjectured that these are all the central configurations of the spatial 5-body problem with equal masses. Alvarez-Ramírez et al. (2008) proved that if there are other central configurations of the 5-body problem with equal masses they must be non-symmetric. Lee and Santoprete (2009) used a computer algebra algorithm, which is devised to find all the isolated solutions of a polynomial system, to find all the isolated central configurations of the 5-body problem with equal masses. They did not find anything more than the central configurations of Kotsireas and Lazard.

Next we describe these four classes of symmetric central configurations given in Kotsireas and Lazard (2002), and we also give the positions $\mathbf{q}_{i}$ and the mutual distances $r_{i j}$ for a representative of each class in the variables that we are working, see Alvarez-Ramírez et al. (2008) for more details. Without loss of generality we assume that $m_{1}=m_{2}=m_{3}=m_{4}=$ $m_{5}=1$.

$K_{1}$ : $(5 \mathrm{cc})$ Four masses located at the vertices of a regular tetrahedron and one mass located at the barycenter, see Fig. 2a.

Positions: $\mathbf{q}_{1}=(0,0,1), \mathbf{q}_{2}=\left(-\sqrt{\frac{2}{3}}, \frac{\sqrt{2}}{3},-\frac{1}{3}\right), \mathbf{q}_{3}=\left(0,-\frac{2 \sqrt{2}}{3},-\frac{1}{3}\right), \mathbf{q}_{4}=$ $\left(\sqrt{\frac{2}{3}}, \frac{\sqrt{2}}{3},-\frac{1}{3}\right), \mathbf{q}_{5}=(0,0,0)$.

Mutual distances: $r_{15}=r_{25}=r_{35}=r_{45}=1, r_{12}=r_{13}=r_{14}=r_{23}=r_{24}=r_{34}=$ $2 \sqrt{\frac{2}{3}}=1.6329931 \ldots$

$K_{2}$ : (15 cc) Five masses located at the vertices of a regular pyramid having a square base, see Fig. $2 b$. 
Fig. 2 Central configurations of the spatial 5-body problem with equal masses. a Class $K_{1}$, b class $K_{3}$, c class $K_{2}$, d class $K_{4}$

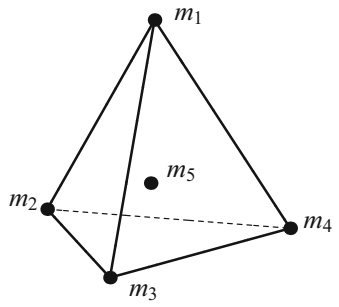

(a)

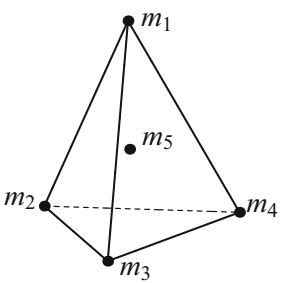

(b)

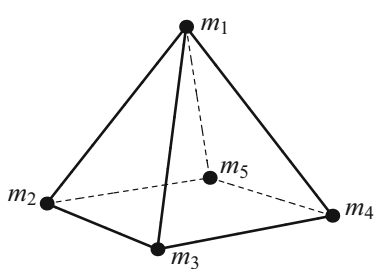

(c)

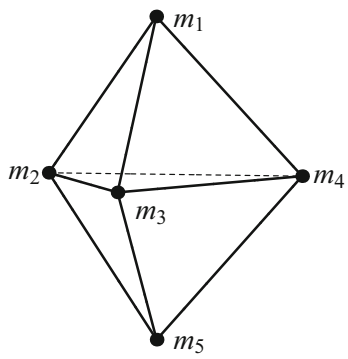

(d)

Positions: $\mathbf{q}_{1}=(0,0,1), \mathbf{q}_{2}=\left(-\frac{5}{\alpha}, 0,-\frac{1}{4}\right), \mathbf{q}_{3}=\left(0,-\frac{5}{\alpha},-\frac{1}{4}\right), \mathbf{q}_{4}=$ $\left(\frac{5}{\alpha}, 0,-\frac{1}{4}\right), \mathbf{q}_{5}=\left(0, \frac{5}{\alpha},-\frac{1}{4}\right)$, where

$$
\alpha=\sqrt{64\left(\frac{2}{7}\right)^{2 / 3} \sqrt[3]{9-4 \sqrt{2}}-16} .
$$

Mutual distances: $r_{12}=r_{13}=r_{14}=r_{15}=1.5944855 \ldots, r_{23}=r_{25}=r_{34}=r_{45}=$ $1.3999173 \ldots, r_{24}=r_{35}=1.9797821 \ldots$.

$K_{3}$ : $(20 \mathrm{cc}$ ) Four masses located at the vertices of a regular pyramid having a base formed by an equilateral triangle and a fifth mass on its axis of symmetry and in the interior of the pyramid, see Fig. 2c.

Positions:

$$
\begin{aligned}
& \mathbf{q}_{1}=(0,0,1), \mathbf{q}_{2}=(-0.71312666 \ldots, 0.41172387 \ldots,-0.38049250 \ldots), \\
& \mathbf{q}_{3}=(0,-0.82344774 \ldots,-0.38049250 \ldots), \\
& \mathbf{q}_{4}=(0.71312666 \ldots, 0.41172387 \ldots,-0.380492504 \ldots), \\
& \mathbf{q}_{5}=(0,0,0.14147751 \ldots) .
\end{aligned}
$$

Mutual distances: $r_{12}=r_{13}=r_{14}=1.6074283 \ldots, r_{25}=r_{35}=r_{45}=$ $0.97494558 \ldots, r_{15}=0.85852248 \ldots, r_{23}=r_{24}=r_{34}=1.4262533 \ldots$.

$K_{4}$ : $(10 \mathrm{cc})$ Three masses located at the vertices of an equilateral triangle and two masses with symmetric positions on the axis orthogonal to the plane defined by the triangle that passes through its barycenter, see Fig. 2d. 
Positions: $\mathbf{q}_{1}=(0,0,1), \mathbf{q}_{2}=(-0.84812349 \ldots, 0.48966432 \ldots, 0), \mathbf{q}_{3}=(0,-0.9$ $7932865 \ldots, 0), \mathbf{q}_{4}=(0.84812349 \ldots, 0.48966432 \ldots, 0), \mathbf{q}_{5}=(0,0,-1)$.

Mutual distances: $r_{12}=r_{13}=r_{14}=r_{25}=r_{35}=r_{45}=1.3996730 \ldots, r_{15}=$ $2, r_{23}=r_{24}=r_{34}=1.6962469 \ldots$

\section{Central configuration in the restricted spatial $(4+1)$-body problem with four equal masses}

The restricted $(4+1)$-body problem is a particular case of the 5-body problem with 4 equal masses plus an infinitesimal mass, where the four particles with positive masses are disposed in a central configuration. From now on, when we say the spatial $(4+1)$-body problem we mean the restricted spatial $(4+1)$-body problem.

It is known that the planar 4-body problem with equal masses has three different classes of central configurations. These consist of a square, an equilateral triangle with one mass at its barycenter, and an isosceles triangle with another mass on its axis of symmetry, see Albouy (1995). However, there exists a unique class of spatial central configurations of the 4-body problem given by the regular tetrahedron, see for instance Lehmann-Filhés (1891).

In the following subsections we shall discuss the central configurations of the spatial $(4+1)$-body problem for each class of central configurations of the planar and spatial 4-body problem with equal masses.

\subsection{Central configurations of the $(4+1)$-body problem with four masses at the vertices of a regular tetrahedron}

Santos (2004) proved that all central configurations of the $(4+1)$-body problem with the four positive equal masses forming a regular tetrahedron have either two planes of symmetry (planar type symmetry), or one axis of symmetry (axis type symmetry). Using these symmetries he found the central configurations of the problem, in particular, he proved that there are 25 central configurations of which 12 are non-convex. Later, Tsai (2012) found the same result using Gröebner bases. These 25 central configurations provide 6 different classes of central configurations. Leandro (2008) gives a different proof of the fact that the spatial central configurations of the restricted $(4+1)$-body problem with equal masses are symmetric.

Next we describe these 6 classes and we give the positions $\mathbf{q}_{i}$ and the mutual distances $r_{i j}$ for a representative of each class in the variables that we are working, see Santos (2004) for more details.

We will assume that $m_{1}=\cdots=m_{4}=1$ and that the positions of the vertices of the tetrahedron are $\mathbf{q}_{1}=(0,0,1), \mathbf{q}_{2}=\left(-\sqrt{\frac{2}{3}}, \frac{\sqrt{2}}{3},-\frac{1}{3}\right), \mathbf{q}_{3}=\left(0,-\frac{2 \sqrt{2}}{3},-\frac{1}{3}\right), \mathbf{q}_{4}=$ $\left(\sqrt{\frac{2}{3}}, \frac{\sqrt{2}}{3},-\frac{1}{3}\right)$. Then we have the following mutual distances

$$
r_{12}=r_{13}=r_{14}=r_{23}=r_{24}=r_{34}=2 \sqrt{\frac{2}{3}} .
$$

$\left(T_{b}\right)$ Axis and planar type symmetry simultaneously: $(1 \mathrm{cc})$ The infinitesimal mass is located at the barycenter of the tetrahedron; that is, $\mathbf{q}_{5}=(0,0,0)$ (see Fig. 3). 
Fig. 3 Central configurations of the spatial $(4+1)$-body problem with four equal masses at the vertices of a regular tetrahedron. a Axis type symmetry, b planar type symmetry

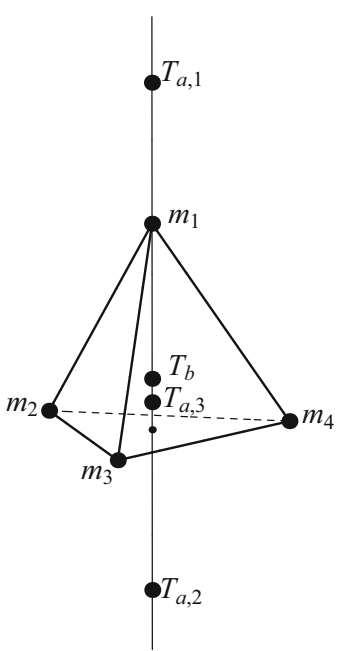

(a)

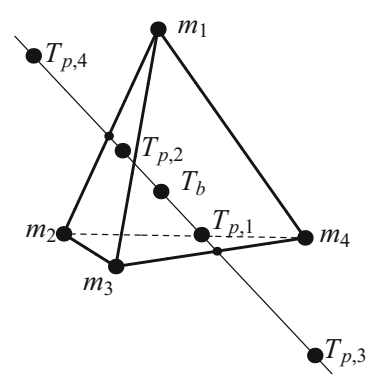

(b)

Mutual distances: $r_{15}=r_{25}=r_{35}=r_{45}=1$.

( $\left.T_{a}\right)$ Axis type symmetry: $(12 \mathrm{cc})$ The infinitesimal mass is located on the line that passes through a vertex and the barycenter of the tetrahedron, see Fig. 3a.

$T_{a, 1}:(4 \mathrm{cc})$ Position of $m_{5}: \mathbf{q}_{5}=(0,0,1.889991 \ldots)$

Mutual distances: $r_{15}=0.88999157 \ldots, r_{25}=r_{35}=r_{45}=2.14149663 \ldots$.

$T_{a, 2}:$ (4 cc) Position of $m_{5}: \mathbf{q}_{5}=(0,0,-1.3981650 \ldots)$

Mutual distances: $r_{15}=2.3981650 \ldots, r_{25}=r_{35}=r_{45}=1.4222360 \ldots$

$T_{a, 3}:$ (4 cc) Position of $m_{5}: \mathbf{q}_{5}=(0,0,-0.1529969 \ldots)$

Mutual distances: $r_{15}=1.1529969 \ldots, r_{25}=r_{35}=r_{45}=0.95990109 \ldots$

$\left(T_{p}\right)$ Planar type symmetry: $(12 \mathrm{cc})$ The infinitesimal mass is located on an edge bisector line passing through the barycenter of the tetrahedron, see Fig. $3 \mathrm{~b}$.

\section{$T_{p, 1}:(6 \mathrm{cc})$}

Position of $m_{5}: \mathbf{q}_{5}=(0.29790401 \ldots,-0.17193722 \ldots,-0.24315596 \ldots)$

Mutual distances: $r_{15}=r_{25}=1.2898396 \ldots, r_{35}=r_{45}=0.83130171 \ldots$..

$T_{p, 3}:(6 \mathrm{cc})$

Position of $m_{5}: \mathbf{q}_{5}=(1.0542556 \ldots,-0.60867481 \ldots,-0.86079617 \ldots)$

Mutual distances: $r_{15}=r_{25}=2.2236237 \ldots, r_{35}=r_{45}=1.2252827 \ldots$

We note that the central configurations $T_{p, 2}$ and $T_{p, 4}$ which appear in Fig. $3 \mathrm{~b}$ belong to the classes $T_{p, 1}$ and $T_{p, 3}$ respectively.

\subsection{Central configurations of the spatial $(4+1)$-body problem with four masses at the vertices of a planar central configuration of the 4-body problem}

Here we provide the coordinates of the bodies for central configurations in the spatial $(4+1)$ body problem with four coplanar equal masses in a central configuration of the 4-body problem. Also, we show the uniqueness (up to rotations and scalings) of this kind of central configurations. 


\subsubsection{Central configurations of the spatial $(4+1)$-body problem with the four equal masses at the vertices of a square}

Fayçal (1996) proved the following result for the pyramidal central configurations of the 5-body problem with a square base. Recall that here a pyramidal central configuration of the 5-body problem means a configuration with four coplanar bodies and a fifth being off the plane.

Theorem 1 (Theorem 4.1 in Fayçal 1996) There exists a unique pyramidal central configuration with a square base, the one of mutual distances

$$
\begin{aligned}
r_{12}=r_{23} & =r_{34}=r_{14}=1, \quad r_{13}=r_{24}=\sqrt{2}, \\
r_{i 5} & =\frac{2}{\sqrt[3]{4+\sqrt{2}}}, \quad i=1, \ldots, 4,
\end{aligned}
$$

where the masses on the base $m_{1}, m_{2}, m_{3}$ and $m_{4}$ are equal, and the mass at the top vertex $m_{5}$ is arbitrary.

Since $m_{5}$ is arbitrary, for $m_{5}=0$ we have the following for the spatial (4+1)-body problem.

Corollary 1 There exists a unique (up to rotations and scalings) central configuration of the spatial $(4+1)$-body problem with four equal masses $m_{1}=m_{2}=m_{3}=m_{4}=1$ in a square and the fifth mass, which is infinitesimal, being off the plane formed by the first four, it is the one with the mutual distances given by (3); or equivalently, the one with positions

$$
\begin{aligned}
& \mathbf{q}_{1}=\left(\frac{1}{2},-\frac{1}{2}, 0\right), \quad \mathbf{q}_{2}=\left(\frac{1}{2}, \frac{1}{2}, 0\right), \quad \mathbf{q}_{3}=\left(-\frac{1}{2}, \frac{1}{2}, 0\right), \\
& \mathbf{q}_{4}=\left(-\frac{1}{2},-\frac{1}{2}, 0\right), \quad \mathbf{q}_{5}=\left(0,0, \sqrt{2\left(\frac{2}{7}\right)^{2 / 3} \sqrt[3]{9-4 \sqrt{2}}-\frac{1}{2}}\right) .
\end{aligned}
$$

\subsubsection{Central configurations of the spatial $(4+1)$-body problem with the coplanar equal masses at the vertices of an equilateral triangle with one mass in its barycenter}

In a recent work Santos and Vidal (2007) proved for the central configurations of the spatial $(4+1)$-body problem that when four equal masses are at the vertices of an equilateral triangle with a mass at the barycenter, the infinitesimal mass must be on a plane of symmetry. In particular, naming the masses in such a way that $m_{1}, m_{2}$, and $m_{3}$ are at the vertices of the triangle, $m_{4}$ is at the barycenter and $m_{5}$ is the infinitesimal mass, they proved that the mutual distances should satisfy either $r_{15}=r_{25}$, or $r_{25}=r_{35}$, or $r_{15}=r_{35}$. However, they do not compute these mutual distances and they do not prove the uniqueness of these classes of central configurations. Since we cannot find in the literature these computations we have calculated them in what follows.

From Theorem 2 in Bang and Elmabsout (2003) we know that the gravitational field generated at the point $\mathbf{q}$ by $n$ equal masses at the vertices of and $n$-gon is directed towards the centre of the $n$-gon if and only if $\mathbf{q}$ belongs to an axis of symmetry of the $n$-gon, or to the axis orthogonal to the plane defined by the $n$-gon that passes through its center. Clearly if there is a mass in the center of the $n$-gon the result remains valid. This means that the infinitesimal mass of the spatial $(4+1)$-body problem, when three of the four equal masses are at the vertices of 
an equilateral triangle and the fourth one is at its barycenter, should be placed on the straight line orthogonal to the plane defined by the triangle that passes through its barycenter.

To compute those central configurations, the most convenient equations are the DziobekLaura-Andoyer equations (see for instance Hampton and Santoprete 2007 and the references therein)

$$
f_{i j h}=\sum_{k \neq i, j, h} m_{k}\left(R_{i k}-R_{j k}\right) \Delta_{i j h k}=0 .
$$

Here $R_{i j}=1 / r_{i j}^{3}$ and $\Delta_{i j h k}=\left(\mathbf{q}_{i}-\mathbf{q}_{j}\right) \wedge\left(\mathbf{q}_{j}-\mathbf{q}_{h}\right) \cdot\left(\mathbf{q}_{h}-q_{k}\right)$ is six times the signed volume of the tetrahedron formed by $m_{i}, m_{j}, m_{h}$ and $m_{k}$. Clearly $R_{i j}=R_{j i}$. Moreover if $P(i, j, h, k)$ is a permutation of the indices $i, j, h, k$, then $\Delta_{P(i, j, h, k)}=\operatorname{sign}(P) \Delta_{i j h k}$ where $\operatorname{sign}(P)$ is the sign of the permutation. In particular $f_{i j h}=f_{j i h}$. Note that in these equations $\Delta_{1234}=0$ because $m_{1}, m_{2}, m_{3}, m_{4}$ are assumed to be coplanar.

We assume that the masses are $m_{1}=m_{2}=m_{3}=m_{4}=1, m_{5}=0$ and that their vector positions are $\mathbf{q}_{1}=(1,0,0), \mathbf{q}_{2}=\left(-\frac{1}{2}, \frac{\sqrt{3}}{2}, 0\right), \mathbf{q}_{3}=\left(-\frac{1}{2},-\frac{\sqrt{3}}{2}, 0\right), \mathbf{q}_{4}=(0,0,0)$ and $\mathbf{q}_{5}=(0,0, z)$. It is easy to check that the following relations are satisfied

$$
r_{12}=r_{23}=r_{13}=\sqrt{3}, \quad r_{14}=r_{24}=r_{34}=1, \quad r_{15}=r_{25}=r_{35} .
$$

Moreover using the symmetry given by the Bang-Elmabsout result and the fact that the volume of a pyramid is the product of the area of the base by the height divided by three, we have the following relations

$$
\Delta_{1245}=-\Delta_{1345}=\Delta_{2345} .
$$

By substituting these values into system (4), it becomes equivalent to the equation

$$
f_{152}=\Delta_{1235}\left(R_{15}-\frac{1}{3 \sqrt{3}}\right)+\Delta_{2345}\left(R_{45}-1\right)=0 .
$$

Therefore

$$
R_{15}=-\frac{\Delta_{2345}}{\Delta_{1235}}\left(R_{45}-1\right)+\frac{1}{3 \sqrt{3}} .
$$

On the other hand it is easy to check that $r_{15}=\sqrt{1+z_{5}^{2}}$ and $r_{45}=z_{5}$. Moreover $\Delta_{1235}=-3 \sqrt{3} z_{5} / 2$ and $\Delta_{2345}=-\sqrt{3} z_{5} / 2$. Then from the relation (5) we get the equation

$$
h=\frac{1}{9}\left(\frac{3}{z_{5}^{3}}+\frac{9}{\left(z_{5}^{2}+1\right)^{3 / 2}}-\sqrt{3}-3\right)=0 .
$$

Here we have assumed that $z,>0$, but this is not restrictive. It is easy to check that

$$
\frac{d h}{d z_{5}}=-\frac{1}{z_{5}^{4}}-\frac{3 z_{5}}{\left(z_{5}^{2}+1\right)^{5 / 2}}<0,
$$

for all $z_{5}>0$. Moreover $h$ is continuous for $z_{5} \in(0,+\infty), \lim _{z_{5} \rightarrow 0^{+}} h=+\infty$ and $\lim _{z_{5} \rightarrow+\infty} h=(-\sqrt{3}-3) / 9$. Therefore Eq. (6) has a unique real solution with $z_{5}>0$, which can be found numerically and it is given by

$$
z_{5}=1.1264766 \ldots
$$

In short we have the following result. 
Theorem 2 There exists a unique class of central configurations of the spatial (4+1)-body problem with four coplanar equal masses, three at the vertices of an equilateral triangle and the fourth at its barycenter. A representative of this class is

$$
\begin{aligned}
& \mathbf{q}_{1}=(1,0,0), \quad \mathbf{q}_{2}=\left(-\frac{1}{2}, \frac{\sqrt{3}}{2}, 0\right), \quad \mathbf{q}_{3}=\left(-\frac{1}{2},-\frac{\sqrt{3}}{2}, 0\right), \quad \mathbf{q}_{4}=(0,0,0), \\
& \mathbf{q}_{5}=(0,0,1.1264766 \ldots)
\end{aligned}
$$

\subsubsection{Central configurations of the spatial $(4+1)$-body problem with the coplanar equal masses at the vertices of an isosceles triangle with one mass on the axis of symmetry}

The configuration of the 4-body problem with three equal masses at the vertices of an isosceles triangle with another equal mass on its axis of symmetry is well known, see Albouy (1995); Llibre (1976). From Llibre (1976) we know that if the base of the nonequal side of the triangle is 2 , then the height of the triangle is $1.81723939472383 \ldots$ and the fourth mass is at a distance from the base equal to $0.6503784729520659 \ldots$...

Assume that $m_{1}=m_{2}=m_{3}=m_{4}=1$ and that the positions of the masses are $\mathbf{q}_{1}=(-1 / 2,-a, 0), \mathbf{q}_{2}=(1 / 2,-a, 0), \mathbf{q}_{3}=(0, c, 0)$ and $\mathbf{q}_{4}=(0, b, 0)$. Since the center of mass is fixed at the origin of coordinates $b=2 a-c$. Then from Llibre (1976) we have that

$$
(a, c)=\left(a^{*}, c^{*}\right)=(0.3084522 \ldots, 0.6001674 \ldots) .
$$

Now we find the central configurations of the spatial (4+1)-body problem with 4 masses equal to 1 at $\mathbf{q}_{1}=\left(-1 / 2,-a^{*}, 0\right), \mathbf{q}_{2}=\left(1 / 2,-a^{*}, 0\right), \mathbf{q}_{3}=\left(0, c^{*}, 0\right)$ and $\mathbf{q}_{4}=\left(0,2 a^{*}-c^{*}, 0\right)$ and an infinitesimal mass at $\mathbf{q}_{5}=\left(x_{5}, y_{5}, z_{5}\right)$. Clearly,

$$
r_{12}=1, \quad r_{13}=r_{23}, \quad r_{14}=r_{24},
$$

and $\Delta_{1234}=0$. By using the symmetry of the isosceles triangle of the base of the pyramid and the fact that the volume of a pyramid is the product of the area of the base by the height divided by three, we get $\Delta_{1345}=-\Delta_{2345}$. Then

$$
f_{354}=\Delta_{2345}\left(R_{15}-R_{25}\right)=0,
$$

so $R_{15}=R_{25}$ because $\Delta_{2345} \neq 0$. Substituting these conditions to (4) we get the system

$$
\begin{aligned}
& f_{1}=\Delta_{1235}\left(1-R_{23}\right)-\Delta_{2345}\left(R_{34}-R_{24}\right)=0, \\
& f_{2}=\Delta_{1245}\left(1-R_{24}\right)-\Delta_{2345}\left(R_{23}-R_{34}\right)=0, \\
& f_{3}=\Delta_{1235}\left(R_{23}-R_{35}\right)+\Delta_{1245}\left(R_{24}-R_{45}\right)=0, \\
& f_{4}=\Delta_{1235}\left(R_{15}-1\right)-\Delta_{2345}\left(R_{24}-R_{45}\right)=0, \\
& f_{5}=\Delta_{1245}\left(R_{15}-1\right)-\Delta_{2345}\left(R_{35}-R_{23}\right)=0 .
\end{aligned}
$$

It is easy to check, by substituting the values of $\mathbf{q}_{i}$, that the first two equations of (7) are always satisfied. 
From condition $R_{25}=R_{15}$ we get $x_{5}=0$. After some computations we get

$$
\begin{aligned}
& \Delta_{1235}=-\left(a^{*}+c^{*}\right) z_{5}, \quad \Delta_{1245}=\left(-3 a^{*}+c^{*}\right) z_{5}, \quad \Delta_{2345}=\left(a^{*}-c^{*}\right) z_{5}, \\
& R_{23}=\frac{1}{\left(\left(a^{*}+c^{*}\right)^{2}+1 / 4\right)^{3 / 2}}, \quad R_{24}=\frac{1}{\left(\left(c^{*}-3 a^{*}\right)^{2}+\frac{1}{4}\right)^{3 / 2}}, \\
& R_{34}=\frac{1}{\left(2\left(c^{*}-a^{*}\right)\right)^{3}}, \quad R_{15}=\frac{1}{r_{15}^{3}}=\frac{1}{\left(\left(a^{*}+y_{5}\right)^{2}+z_{5}^{2}+1 / 4\right)^{3 / 2}}, \\
& R_{35}=\frac{1}{r_{35}^{3}}=\frac{1}{\left(\left(c^{*}-y_{5}\right)^{2}+z_{5}^{2}\right)^{3 / 2}}, \quad R_{45}=\frac{1}{r_{45}^{3}}=\frac{1}{\left(\left(2 a^{*}-c^{*}-y_{5}\right)^{2}+z_{5}^{2}\right)^{3 / 2}} .
\end{aligned}
$$

Since we are not interested in the solutions of (7) with $z_{5}=0$, we can consider the system of equations $\bar{f}_{3}=f_{3} / z_{5}=0$, and $\bar{f}_{4}=f_{4} / z_{5}=0$, where

$$
\begin{aligned}
& \bar{f}_{3}\left(y_{5}, z_{5}\right)=-\left(a^{*}+c^{*}\right) R_{23}-\left(3 a^{*}-c^{*}\right) R_{24}+\frac{a^{*}+c^{*}}{r_{35}^{3}}+\frac{3 a^{*}-c^{*}}{r_{45}^{3}}, \\
& \bar{f}_{4}\left(y_{5}, z_{5}\right)=a^{*}+c^{*}-\left(a^{*}-c^{*}\right) R_{24}-\frac{a^{*}+c^{*}}{r_{15}^{3}}+\frac{a^{*}-c^{*}}{r_{45}^{3}}=0 .
\end{aligned}
$$

We note that $a^{*}+c^{*}>0, a^{*}-c^{*}<0$ and $3 a^{*}-c^{*}>0$.

Next we analyze the functions $\bar{f}_{3}$ and $\bar{f}_{4}$. Notice that $r_{15}$ is always different from zero $r_{35}=0$ when $\left(y_{5}, z_{5}\right)=\left(c^{*}, 0\right)$ and $r_{45}=0$ when $\left(y_{5}, z_{5}\right)=\left(2 a^{*}-c^{*}, 0\right)$.

It is easy to check that the analytic function $\bar{f}_{4}$ is defined in $D_{4}=\mathbb{R}^{2} \backslash\left\{\left(2 a^{*}-c^{*}, 0\right)\right\}$, that it has no critical points, and that $\lim _{\left(y_{5}, z_{5}\right) \rightarrow(2 a-c, 0)} \bar{f}_{4}\left(y_{5}, z_{5}\right)=-\infty$. Therefore all the level curves of $\bar{f}_{4}$ consist of a unique single closed curve. Since $a^{*}+c^{*}>0$ and $a^{*}-c^{*}<0$, $\bar{f}_{4}\left(y_{5}, z_{5}\right)<a^{*}+c^{*}-\left(a^{*}-c^{*}\right) R_{24}=2.283438 \ldots$ for all $\left(y_{5}, z_{5}\right) \in D$, then we can assure that the level curve $\bar{f}_{4}\left(y_{5}, z_{5}\right)=0$ is a single closed curve surrounding the point $\left(2 a^{*}-c^{*}, 0\right)$.

The function $\bar{f}_{3}$ is analytic in $D_{3}=\mathbb{R}^{2} \backslash\left\{\left(2 a^{*}-c^{*}, 0\right) \cup\left(c^{*}, 0\right)\right\}$ and it satisfies that $\bar{f}_{3}\left(y_{5}, z_{5}\right)>-\left(a^{*}+c^{*}\right) R_{23}-\left(3 a^{*}-c^{*}\right) R_{24}=-2.3471168 \ldots$ for all $\left(y_{5}, z_{5}\right) \in D_{3}$. Moreover $\lim _{\left(y_{5}, z_{5}\right) \rightarrow\left(2 a^{*}-c^{*}, 0\right)} \bar{f}_{3}\left(y_{5}, z_{5}\right)=+\infty$ and $\lim _{\left(y_{5}, z_{5}\right) \rightarrow\left(c^{*}, 0\right)} \bar{f}_{3}\left(y_{5}, z_{5}\right)=+\infty$. Then the level curves $\bar{f}_{3}\left(y_{5}, z_{5}\right)=K$ for $K$ sufficiently large consist of two closed curves, one surrounding the point $\left(2 a^{*}-c^{*}, 0\right)$ and the other one surrounding the point $\left(c^{*}, 0\right)$, because the function $\bar{f}_{3}$ has a unique critical point $\alpha=(0.27118903 \ldots, 0)$ which is a saddle with $\left.\bar{f}_{3}\right|_{\left(y_{5}, z_{5}\right)=\alpha}=\beta=42.911529 \ldots$ More precisely, the curves $\bar{f}_{3}\left(y_{5}, z_{5}\right)=K$ for $K>\beta$ consist of two closed curves that join in a single curve with two loops when $K=\beta$, and became a single closed curve when $K<\beta$. Since $\bar{f}_{3}\left(y_{5}, z_{5}\right)>-2.3471168 \ldots$ for all $\left(y_{5}, z_{5}\right) \in D_{3}$ we can assure that the level curve $\bar{f}_{3}\left(y_{5}, z_{5}\right)=0$ is a single closed curve surrounding the points $\left(2 a^{*}-c^{*}, 0\right)$ and $\left(c^{*}, 0\right)$.

In short, we have proved that each of the level curves $\bar{f}_{3}\left(y_{5}, z_{5}\right)=0$ and $\bar{f}_{4}\left(y_{5}, z_{5}\right)=0$ consist of a single closed curve. We plot these two closed curves with the help of Mathematica in Fig. 4. We see that they intersect at two points which are symmetric with respect to the $y_{5}$ axis. We compute them numerically and we get

$$
\left(y_{5}, z_{5}\right)=(-0.0030046492 \ldots, \pm 0.66658830 \ldots) .
$$

These solutions satisfy the remaining equation $f_{5}=0$ so they provide spatial central configurations of the $(4+1)$-body problem with four equal masses. Notice that, due to the symmetry, these solutions belong to the same class.

This provides proof of the following numerical result. 
Fig. 4 The level curves $\bar{f}_{3}\left(y_{5}, z_{5}\right)=0$ (continuous line) and $\bar{f}_{4}\left(y_{5}, z_{5}\right)=0($ dashed line $)$

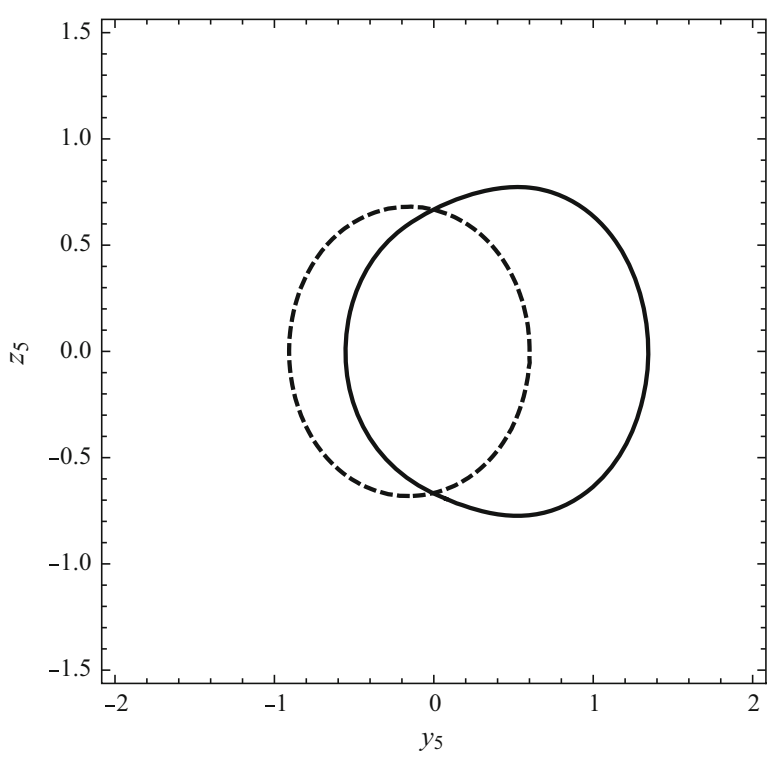

Result 1 We have a unique class of spatial central configurations of the $(4+1)$-body problem with the four equal masses in a central configuration of the 4-body problem with three masses $m_{1}, m_{2}, m_{3}$ at the vertices of an isosceles triangle and one mass $m_{4}$ on its axis of symmetry. A representative of this class is the configuration given $\mathbf{q}_{1}=\left(-1 / 2,-a^{*}, 0\right), \mathbf{q}_{2}=\left(1 / 2,-a^{*}, 0\right), \mathbf{q}_{3}=\left(0, c^{*}, 0\right)$ and $\mathbf{q}_{4}=\left(0,2 a^{*}-c^{*}, 0\right)$ and $\mathbf{q}_{5}=(0,-0.0030046492 \ldots, 0.66658830 \ldots)$.

\subsubsection{Summary of results}

Next we give the positions $\mathbf{q}_{i}$ and the mutual distances $r_{i j}$ for a representative of each class of central configurations of the spatial $(4+1)$-body problem with four equal masses in a coplanar central configurations satisfying the assumptions on $\mathbf{q}_{i}$ made in Sect. 2. We also assume that $m_{1}=\cdots=m_{4}=1$.

$P_{s}:(6 \mathrm{cc})$ The four equal masses are located at the vertices of a square and the infinitesimal mass at the straight line passing through the barycenter of the square and perpendicular to it, see Fig. 5a.

Positions: $\mathbf{q}_{1}=(0,0,1), \mathbf{q}_{2}=(1,0,0), \mathbf{q}_{3}=(0,0,-1), \mathbf{q}_{4}=(-1,0,0), \mathbf{q}_{5}=$ $\left(0, \sqrt{-1+4\left(\frac{2}{7}\right)^{2 / 3} \sqrt[3]{9-4 \sqrt{2}}}, 0\right)$.

Mutual distances: $r_{12}=r_{14}=r_{23}=r_{34}=\sqrt{2}=1.4142135 \ldots, r_{13}=r_{24}=$ $2, r_{15}=r_{25}=r_{35}=r_{45}=2 \sqrt[3]{\frac{2}{7}(2 \sqrt{2}-1)}=1.6107687 \ldots$

Recall that in all configurations we have assumed that $\mathbf{q}_{1}=(0,0,1)$ and that $x_{3}=0$ (see Sect. 2). For this particular configuration, these conditions are satisfied only when the mass $m_{3}$ is located on the $z$-axis. So in this case to avoid rotations of the configuration we should fix for instance $y_{4}=0$ instead of $x_{3}=0$.

$P_{e t}:(24 \mathrm{cc})$ Three masses are located at the vertices of an equilateral triangle with one mass at its barycenter and the infinitesimal mass at the straight line passing through the barycenter of the triangle and perpendicular to it, see Fig. 5 b. 
Fig. 5 Central configurations of the spatial $(4+1)$-body problem with four equal masses at the vertices of a planar central configuration. a Class $P_{S}$, b class $P_{\text {et }}, \mathbf{c}$ class $P_{i t}$

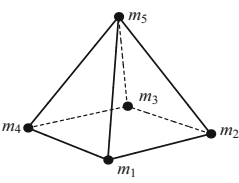

(a)

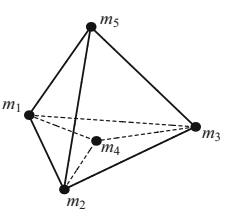

(b)

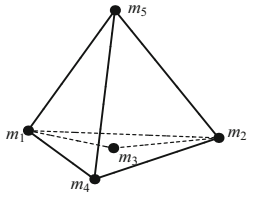

(c)

Positions: $\mathbf{q}_{1}=(0,0,1), \mathbf{q}_{2}=\left(0,-\frac{\sqrt{3}}{2},-\frac{1}{2}\right), \mathbf{q}_{3}=\left(0, \frac{\sqrt{3}}{2},-\frac{1}{2}\right), \mathbf{q}_{4}=(0,0,0), \mathbf{q}_{5}=$ $(1.1264766 \ldots, 0,0)$.

Mutual distances: $r_{12}=r_{13}=r_{23}=\sqrt{3}=1.7320508 \ldots, r_{14}=r_{24}=r_{34}=$ $1, r_{15}=r_{25}=r_{35}=1.5063032, r_{45}=1.1264766 \ldots$

$P_{i t}$ : $(24 \mathrm{cc})$ Three masses located at the vertices of an isosceles triangle with one mass on its axis of symmetry and the infinitesimal mass on the plane perpendicular to the plane of the triangle that contains its axis of symmetry, see Fig. 5c.

Positions: $\mathbf{q}_{1}=(0,0,1), \mathbf{q}_{2}=(0,-0.89369458 \ldots,-0.44867581 \ldots), \mathbf{q}_{3}=$ $(0,0.024246491 \ldots,-0.014957768 \ldots), \mathbf{q}_{4}=(0,0.86944809 \ldots,-0.53636641 \ldots)$, $\mathbf{q}_{5}=(-1.1346405 \ldots,-0.0043527627 \ldots, 0.0026852387 \ldots)$.

Mutual distances: $r_{12}=1.7021608 \ldots, r_{13}=r_{23}=1.0152473 \ldots, r_{14}=$ $r_{24}=1.7653219 \ldots, r_{34}=0.99309250 \ldots, r_{15}=r_{25}=1.5106504 \ldots, r_{35}=$ $1.1351380 \ldots$,

$r_{45}=1.5302005 \ldots$

\section{Analytic continuation of the central configurations}

In this section we continue numerically the families of central configurations from the spatial $(4+1)$-body problem with 4 equal masses to the spatial 5-body problem with equal masses, and vice versa considering one of the masses $m$ as a parameter. In Sect. 2, assuming that $m_{2} \neq 0$, we have reduced the equations of central configurations to a set of eleven equations $e_{i}=0$ with $i=1, \ldots, 11$ and eight unknowns, namely $y_{3}, z_{3}, x_{4}, y_{4}, z_{4}, x_{5}, y_{5}, z_{5}$. So we should find numerically the solutions of that set of eleven equations as $m$ varies from 0 to 1 or vice versa.

Let

$$
M=\left(\begin{array}{cccccccc}
\frac{\partial e_{1}}{\partial y_{3}} & \frac{\partial e_{1}}{\partial z_{3}} & \frac{\partial e_{1}}{\partial x_{4}} & \frac{\partial e_{1}}{\partial y_{4}} & \frac{\partial e_{1}}{\partial z_{4}} & \frac{\partial e_{1}}{\partial x_{5}} & \frac{\partial e_{1}}{\partial y_{5}} & \frac{\partial e_{1}}{\partial z_{5}} \\
\frac{\partial e_{2}}{\partial y_{3}} & \frac{\partial e_{2}}{\partial z_{3}} & \frac{\partial e_{2}}{\partial x_{4}} & \frac{\partial e_{2}}{\partial y_{4}} & \frac{\partial e_{2}}{\partial z_{4}} & \frac{\partial e_{2}}{\partial x_{5}} & \frac{\partial e_{2}}{\partial y_{5}} & \frac{\partial e_{2}}{\partial z_{5}} \\
\ldots & \ldots & \ldots & \ldots & \ldots & \ldots & \ldots & \ldots \\
\frac{\partial e_{11}}{\partial y_{3}} & \frac{\partial e_{11}}{\partial z_{3}} & \frac{\partial e_{11}}{\partial x_{4}} & \frac{\partial e_{11}}{\partial y_{4}} & \frac{\partial e_{11}}{\partial z_{4}} & \frac{\partial e_{11}}{\partial x_{5}} & \frac{\partial e_{11}}{\partial y_{5}} & \frac{\partial e_{11}}{\partial z_{5}}
\end{array}\right) .
$$

We say that a central configuration given by $\mathbf{z}^{0}=\left(y_{3}^{0}, z_{3}^{0}, x_{4}^{0}, y_{4}^{0}, z_{4}^{0}, x_{5}^{0}, y_{5}^{0}, z_{5}^{0}\right)$ for a fixed value of $m=m^{0}$ is degenerate if the rank of the matrix $M$ is not maximal at $m^{0}$ and $\mathbf{z}^{0}$. From the Implicit Function Theorem every non-degenerate central configuration can be continued to a unique family of central configurations as the parameter $m$ varies. As a consequence of the Implicit Function Theorem, the number of central configurations can only change if the degeneracy condition is fulfilled. 
Table 1 For each representative of the classes of central configurations given in Sect. 3 and 4, except for the class $P_{S}$, we provide the values of the minors of order 8 different from zero, and the equations that we have used for computing these minors

\begin{tabular}{|c|c|c|}
\hline Configuration & $\left|M_{e_{s_{i}}}\right|$ & Equations $e_{s_{i}}$ \\
\hline$K_{1}$ & $-3.8792768 \ldots$ & \\
\hline$K_{2}$ & $-0.22353716 \ldots$ & \\
\hline$K_{3}$ & $106.13353 \ldots$ & \\
\hline$K_{4}$ & $0.38988999 \ldots$ & \\
\cline { 1 - 2 }$T_{b}$ & $0.18040672 \ldots$ & \multirow{3}{*}{$e_{1}, e_{2}, e_{3}, e_{4}, e_{5}, e_{6}, e_{8}, e_{11}$} \\
\hline$T_{a, 1}$ & $0.41952832 \ldots$ & \\
\hline$T_{a, 2}$ & $0.099424864 \ldots$ & \\
\hline$T_{a, 3}$ & $-0.59862385 \ldots$ & \\
\hline$T_{p, 1}$ & $5.27579804 \ldots$ & \\
\hline$T_{p, 3}$ & $-0.13695605 \ldots$ & \\
\hline$P_{e t}$ & $-0.080517418 \ldots$ & \multirow{2}{*}{$e_{1}, e_{4}, e_{5}, e_{6}, e_{7}, e_{8}, e_{9}, e_{11}$} \\
\hline$P_{i t}$ & $-0.01076149028 \ldots$ & \\
\hline & &
\end{tabular}

In order to continue the central configurations from the (4+1)-body problem to the 5-body problem or vice versa, we have used the following methodology. First, for each representative of the classes of central configurations given in Sect. 3 and 4, we choose a subset of eight equations $e_{s_{i}}=0$ with $s_{i} \in\{1, \ldots, 11\}$ for $i=1, \ldots, 8$ such that the associated minor of order 8 of the matrix $M$, denoted by $\left|M_{e_{s i}}\right|$, evaluated on that representative does not vanish (see Table 1). This can be done for all the classes except $P_{s}$ because the matrix $M$ evaluated at the representative of the class $P_{s}$ has rank 7 . This is due to the fact that the representative of the class $P_{s}$ satisfying conditions $\mathbf{q}_{1}=(0,0,1)$ and $x_{3}=0$ has the mass $m_{3}$ on the $z$-axis. Thus any rotation of this configuration satisfies also conditions $\mathbf{q}_{1}=(0,0,1)$ and $x_{3}=0$ and consequently it provides a solution of system $e_{s_{i}}=0$ making the configuration degenerate. In this case, we will avoid this degeneracy by working with the new system of equations $\widetilde{e}_{i}=0$ instead of $e_{i}=0$, where the new equations $\widetilde{e}_{i}$ are computed as in Sect. 2 but fixing the variable $y_{4}=0$ instead of $x_{3}=0$. The new Jacobian Matrix $\widetilde{M}$ evaluated at the representative of the class $P_{s}$ has rank 8 . In particular, the subset of equations $\widetilde{e}_{1}, \widetilde{e}_{3}, \widetilde{e}_{4}$, $\widetilde{e}_{5}, \widetilde{e}_{8}, \widetilde{e}_{9}, \widetilde{e}_{10}, \widetilde{e}_{11}$ has an associated determinant $\left|M_{\tilde{e}_{s i}}\right|$ equal to $0.13603300 \ldots \neq 0$.

Once chosen the appropriate subset of equations $e_{s_{i}}=0$ we continue numerically the solution of the system $e_{s_{i}}=0$ from $m=0$ (respectively $m=1$ ), either to $m=1$ (respectively $m=0$ ), or to a value $m^{*}$ such that the determinant $\left|M_{e_{s_{i}}}\right|$ evaluated at the corresponding solution becomes 0 . The continuation method is based in the Newton's algorithm for finding zeros of a vectorial function. We must take some care for finding the values $m^{*}$ according to the parity of the multiplicity of the zeros of $\left|M_{e_{s_{i}}}\right|$. Later on we will specify this parity for the different zeros of the determinant at $m^{*}$.

Finally we check that the solutions of the system $e_{s_{i}}=0$ that we have obtained satisfy the remaining three equations $e_{i}$. In the following we describe the results obtained.

\subsection{Continuation from the $(4+1)$-body problem to the 5 -body problem}

$T_{b}$ : The representative of the class $T_{b}$ can be continued up to $m=m_{c}$ with $m_{c}=$ $\frac{10368+1701 \sqrt{6}}{54952}=0.26449596 \ldots$ through the family of central configurations with 
$\mathbf{q}_{1}(m)=\mathbf{q}_{1}^{c}=(0,0,1), \mathbf{q}_{2}(m)=\mathbf{q}_{2}^{c}=\left(-\sqrt{\frac{2}{3}}, \frac{\sqrt{2}}{3},-\frac{1}{3}\right), \mathbf{q}_{3}(m)=\mathbf{q}_{3}^{c}=$ $\left(0,-\frac{2 \sqrt{2}}{3},-\frac{1}{3}\right), \mathbf{q}_{4}(m)=\mathbf{q}_{4}^{c}=\left(\sqrt{\frac{2}{3}}, \frac{\sqrt{2}}{3},-\frac{1}{3}\right)$, and $\mathbf{q}_{5}(m)=\mathbf{q}_{5}^{c}=(0,0,0)$ which consists of a regular tetrahedron with four masses equal to 1 and the variable mass at its barycenter. The value $m_{c}$ corresponds to the degenerate central configuration found by Schmidt (1988) and Santos (2004). The parity of $m_{c}$ as a zero of $\left|M_{e_{s_{i}}}\right|$ is odd.

From now on we will denote by $q_{c}$ the degenerate configuration with $\left(\mathbf{q}_{1}, \mathbf{q}_{2}, \mathbf{q}_{3}\right.$, $\left.\mathbf{q}_{4}, \mathbf{q}_{5}\right)=\left(\mathbf{q}_{1}^{c}, \mathbf{q}_{2}^{c}, \mathbf{q}_{3}^{c}, \mathbf{q}_{4}^{c}, \mathbf{q}_{5}^{c}\right)$ and $m=m_{c}$.

$T_{a, 1}$ : The representative of the class $T_{a, 1}$ can be continued to a family that ends when $m=1$ at a central configuration of mutual distances

$$
\begin{array}{ll}
r_{12}=r_{13}=r_{14}=\alpha(0.97494558 \ldots), & r_{23}=r_{24}=r_{34}=\alpha(1.4262533 \ldots), \\
r_{25}=r_{35}=r_{45}=\alpha(1.6074283 \ldots), & r_{15}=\alpha(0.85852248 \ldots),
\end{array}
$$

where $\alpha=7.0682611 \ldots$ This configuration corresponds to the representative of class $K_{3}$ scaled by a factor $\alpha$ and with the positions of the masses $m_{1}$ and $m_{5}$ interchanged. All the configurations of this family satisfy the symmetry $r_{12}=r_{13}=r_{14}, r_{23}=r_{24}=r_{34}$, $r_{25}=r_{35}=r_{45}$.

$T_{a, 2}$ : The representative of the class $T_{a, 2}$ can be continued to a family that ends when $m=1$ at a central configuration of mutual distances

$$
\begin{aligned}
& r_{12}=r_{13}=r_{14}=r_{25}=r_{35}=r_{45}=1.3999673 \ldots, \quad r_{15}=2, \\
& r_{23}=r_{24}=r_{34}=1.6962469 \ldots
\end{aligned}
$$

This configuration corresponds to the representative of class $K_{4}$. All the configurations of this family satisfy the symmetry $r_{12}=r_{13}=r_{14}, r_{23}=r_{24}=r_{34}, r_{25}=r_{35}=r_{45}$.

$T_{a, 3}$ : The representative of the class $T_{a, 3}$ can be continued to a family that ends at the degenerate configuration $q_{c}$. Here the parity of $m_{c}$ as zero of $\left|M_{e_{s_{i}}}\right|$ is even. All the configurations of this family satisfy the symmetry $r_{12}=r_{13}=r_{14}, r_{23}=r_{24}=r_{34}$, $r_{25}=r_{35}=r_{45}$.

$T_{p, 1}$ : The representative of the class $T_{p, 1}$ can be continued to a family that ends at the degenerate configuration $q_{c}$. The parity of $m_{c}$ as zero of $\left|M_{e_{s_{i}}}\right|$ is odd. All the configurations of this family satisfy the symmetry $r_{13}=r_{14}=r_{23}=r_{24}, r_{15}=r_{25}, r_{35}=r_{45}$.

$T_{p, 3}$ : The representative of the class $T_{p, 3}$ can be continued to a family that ends at a degenerate configuration with $m=m_{f}=0.66345045 \ldots$ and with position vectors

$$
\begin{aligned}
& \mathbf{q}_{1}=\mathbf{q}_{1}^{f}=(0,0,1), \\
& \mathbf{q}_{2}=\mathbf{q}_{2}^{f}=(-0.89217754 \ldots, 0.12568104 \ldots,-0.43384732 \ldots), \\
& \mathbf{q}_{3}=\mathbf{q}_{3}^{f}=(0,-0.99969077 \ldots,-0.087625913 \ldots), \\
& \mathbf{q}_{4}=\mathbf{q}_{4}^{f}=(0.27617240 \ldots, 0.96078637 \ldots,-0.087625913 \ldots), \\
& \mathbf{q}_{5}=\mathbf{q}_{5}^{f}=(0.92848703 \ldots,-0.13079596 \ldots,-0.58919373 \ldots),
\end{aligned}
$$

see Fig. 6. The corresponding mutual distances are

$$
\begin{array}{lll}
r_{13}=r_{14}=r_{23}=r_{24}=1.47726496 \ldots, & & r_{15}=r_{25}=1.84519172 \ldots, \\
r_{35}=r_{45}=1.36698085 \ldots, & & r_{12}=1.69342689 \ldots, \\
r_{34}=1.97983379 \ldots & &
\end{array}
$$

The parity of $m_{f}$ as zero of $\left|M_{e_{s_{i}}}\right|$ is odd. All the configurations of this family satisfy the symmetry $r_{13}=r_{14}=r_{23}=r_{24}, r_{15}=r_{25}, r_{35}=r_{45}$.

From now on we will denote by $q_{f}$ the class of degenerate central configurations with representative $\left(\mathbf{q}_{1}, \mathbf{q}_{2}, \mathbf{q}_{3}, \mathbf{q}_{4}, \mathbf{q}_{5}\right)=\left(\mathbf{q}_{1}^{f}, \mathbf{q}_{2}^{f}, \mathbf{q}_{3}^{f}, \mathbf{q}_{4}^{f}, \mathbf{q}_{5}^{f}\right)$ and $m=m_{f}$. 
Fig. 6 Configuration $q_{f}$ corresponding to $m=m_{f}$

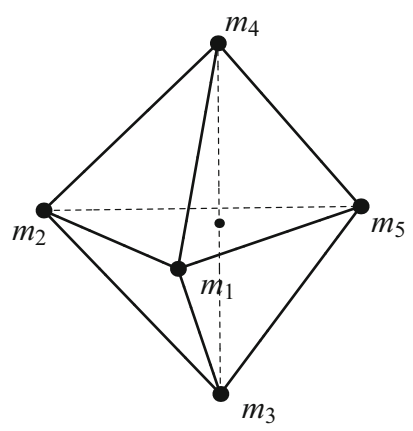

Notice that the bifurcation point $m_{f}$ shows a bifurcation inside the set of convex spatial central configurations, while it is conjectured that in the planar 4-body problem there is no bifurcation inside the set of convex planar central configurations, see for instance Albouy and Fu (2007).

$P_{s}$ : The representative of the class $P_{s}$ can be continued to a family ending at $m=1$ with the configuration of mutual distances

$$
\begin{aligned}
& r_{12}=r_{14}=r_{23}=r_{34}=\alpha(1.39991735 \ldots), \quad r_{13}=r_{24}=\alpha(1.97978210 \ldots), \\
& r_{15}=r_{25}=r_{35}=r_{45}=\alpha(1.59448559 \ldots),
\end{aligned}
$$

with $\alpha=0.97945856 \ldots$. This configuration corresponds to the representative of the class $K_{2}$ scaled by the factor $\alpha$ and with the masses $m_{1}$ and $m_{5}$ interchanged. We note that the central configurations of this family for all the values of $m$ between 0 and 1 correspond to the pyramidal configuration provided by Fayçal (see Theorem 1). The changes in the size of the configuration as $m$ varies are due to the fact that the position of $m_{1}$ is fixed at $(0,0,1)$ and also to the fact that the center of mass of the configuration is fixed at the origin.

$P_{e t}$ : The representative of the class $P_{e t}$ can be continued to a family ending at $m=1$ with the configuration of mutual distances

$$
\begin{aligned}
& r_{12}=r_{13}=r_{15}=r_{23}=r_{25}=r_{35}=1.63299316 \ldots, \\
& r_{14}=r_{24}=r_{34}=r_{45}=1 .
\end{aligned}
$$

This configuration corresponds to the representative of the class $K_{1}$ with the masses $m_{4}$ and $m_{5}$ interchanged. All the configurations of this family satisfy the symmetry $r_{12}=r_{13}=r_{23}, r_{14}=r_{24}=r_{34}, r_{15}=r_{25}=r_{35}$.

$P_{i t}$ : The representative of the class $P_{i t}$ can be continued to a family ending at $m=1$ with the configuration of mutual distances

$$
\begin{array}{ll}
r_{12}=r_{15}=r_{25}=\alpha(1.42625333 \ldots), & r_{13}=r_{23}=r_{35}=\alpha(0.97494558 \ldots), \\
r_{14}=r_{24}=r_{45}=\alpha(1.60742830 \ldots), & r_{34}=\alpha(0.85852248 \ldots),
\end{array}
$$

with $\alpha=1.10240737 \ldots$. This configuration corresponds to the representative of the class $K_{3}$ by interchanging the masses $m_{1}$ and $m_{4}$ and the masses $m_{3}$ and $m_{5}$. All the configurations of this family satisfy the symmetry $r_{13}=r_{23}, r_{14}=r_{24}, r_{15}=r_{25}$. 


\subsection{Continuation from the 5-body problem to the $(4+1)$-body problem}

We only analyze the continuation of the representatives of the classes of central configurations $K_{i}$ that can provide different classes of central configurations of the 5-body problem with 4 equal masses. Since all the central configurations $K_{i}$ are non-degenerate, from the Implicit Function Theorem, each central configuration can be continued to a unique family of central configurations. So the families that have already been obtained in Sect. 5.1 from continuation of central configurations of the $(4+1)$-body problem are not described here.

\subsubsection{Continuation from the class $K_{1}$}

Due to the symmetry of the configuration it is sufficient to analyze the families with $m_{5} \rightarrow 0$ and $m_{1} \rightarrow 0$.

- $\left(m_{5} \rightarrow 0\right)$ The representative of the class $K_{1}$ can be continued to a family that ends at the degenerate configuration $q_{c}$. The parity of $m_{c}$ as zero of $\left|M_{e_{s_{i}}}\right|$ is odd. All the configurations of this family consists of a regular tetrahedron with four masses equal to 1 and the variable mass at its barycenter.

- $\left(m_{1} \rightarrow 0\right)$ The representative of the class $K_{1}$ can be continued to a family ending at $m_{1}=0$ with a representative of the class $P_{e t}$ (see Sect. 5.1).

\subsubsection{Continuation from the class $K_{2}$}

Due to the symmetry of the configuration it is sufficient to analyze the families with $m_{5} \rightarrow 0$ and $m_{1} \rightarrow 0$.

- $\left(m_{5} \rightarrow 0\right)$ The representative of the class $K_{2}$ can be continued to a family that ends at a representative of the degenerate class $q_{f}$. The parity of $m_{f}$ as zero of $\left|M_{e_{s_{i}}}\right|$ is odd. All the configurations of this family satisfy the symmetry $r_{12}=r_{14}, r_{23}=r_{34}=r_{25}=r_{45}$.

- $\left(m_{1} \rightarrow 0\right)$ The representative of the class $K_{2}$ can be continued to a family ending at $m_{1}=0$ with a representative of the class $P_{S}$ (see Sect.5.1).

\subsubsection{Continuation from the class $K_{3}$}

In this case we should analyze the families with $m_{5} \rightarrow 0, m_{1} \rightarrow 0$ and $m_{3} \rightarrow 0$.

- $\left(m_{5} \rightarrow 0\right)$ The representative of the class $K_{3}$ can be continued to a family that ends at the degenerate configuration $q_{c}$. Here the parity of $m_{c}$ as zero of $\left|M_{e_{s_{i}}}\right|$ is even. All the configurations of this family satisfy the symmetry $r_{12}=r_{13}=r_{14}, r_{23}=r_{24}=r_{34}$, $r_{25}=r_{35}=r_{45}$.

- $\left(m_{1} \rightarrow 0\right)$ The representative of the class $K_{3}$ can be continued to a family ending at $m_{1}=0$ with a representative of the class $T_{a, 1}$ (see Sect. 5.1).

- $\left(m_{3} \rightarrow 0\right)$ The representative of the class $K_{3}$ can be continued to a family ending at $m_{3}=0$ with a representative of the class $P_{i t}$ (see Sect.5.1).

\subsubsection{Continuation form the class $K_{4}$}

Due to the symmetry of the configuration it is sufficient to analyze the families with $m_{5} \rightarrow 0$ and $m_{3} \rightarrow 0$. 
- $\left(m_{5} \rightarrow 0\right)$ The representative of the class $K_{4}$ can be continued to a family ending at $m_{5}=0$ with a representative of the class $T_{a, 2}$ (see Sect. 5.1).

- $\left(m_{3} \rightarrow 0\right)$ The representative of the class $K_{4}$ can be continued to a family that ends at a representative of the degenerate class $q_{f}$. The parity of $m_{f}$ as zero of $\left|M_{e_{s_{i}}}\right|$ is odd. All the configurations of this family satisfy the symmetry $r_{12}=r_{14}=r_{25}=r_{45}, r_{13}=r_{35}$, $r_{23}=r_{34}$.

\section{Summary of the main results}

We have proved in Sect. 5 that all the central configurations of the spatial $(4+1)$-body problem with 4 equal masses and all the symmetric central configurations of the spatial 5-body problem with equal masses are non-degenerate. Applying the Implicit Function Theorem we can guarantee that there is a unique family of central configurations parameterized by the nonequal mass $m$ emanating from each non-degenerate configuration. In short we have the following result.

\section{Theorem 3 The following statements hold.}

(a) Let $q_{0}$ be a central configuration of the spatial $(4+1)$-body problem with four equal masses in a central configuration of the 4-body problem. Then, for $m>0$ sufficiently small, the central configuration $q_{0}$ can be analytically continued to a unique family of central configurations of the 5-body problem with four masses equal to one and the fifth mass equal to $m$.

(b) Let $q_{1}$ be a symmetric central configuration of the spatial 5-body problem with five masses equal to one. Then, for $\varepsilon$ sufficiently small, and for each choice of the mass $m_{i}$, the central configuration $q_{1}$ can be analytically continued to a unique family $q_{m}$ of central configurations of the 5-body problem with four masses equal to 1 and the fifth mass $m_{i}=1-\varepsilon$.

We emphasize that to complete the description of the spatial central configurations of the 5 -body problem with four masses equal to one and the fifth mass varying in $[0,1]$ that come from central configurations of the spatial $(4+1)$-body problem with four equal masses it is sufficient to consider the families of central configurations that are different up to rotations and scalings. In Sect. 5 we have continued numerically a representative of each class of the families of central configurations provided by Theorem 3 either from $m=0$ to $m=1$ (or vice versa) or from $m=0$ (or $m=1$ ) to a value of $m$ giving a degenerate central configuration. The results that we have obtained are summarized in Fig. 7. Since there does not exist spatial central configurations at the boundary between convex and non-convex central configurations (see Proposition 12 in Albouy 2003), the results can be divided into two disconnected diagrams, one for convex and one for concave central configurations, see Fig. 7. Notice that all the families of central configurations that we have obtained are symmetric.

In particular, we have proved the following numerical result.

Result 2 The following statements hold for the 5-body problem with four equal masses when the non-equal mass takes values in the interval $[0,1]$.

(a) There exist two (up to rotations and scalings) degenerate central configurations, the one corresponding to the nonequal mass values $m=m_{c}=\frac{10368+1701 \sqrt{6}}{54952}=0.26449596 \ldots$ and $m=m_{f}=0.66345045 \ldots$.. 
Fig. 7 Connections between the classes of central configurations of the $(4+1)$-body problem with 4 equal masses and the ones of the spatial 5-body problem with equal masses. Each line corresponds to a different family of central configurations of the 5-body problem with 4 equal masses. The black dots represent mass values where degenerate central configurations occur

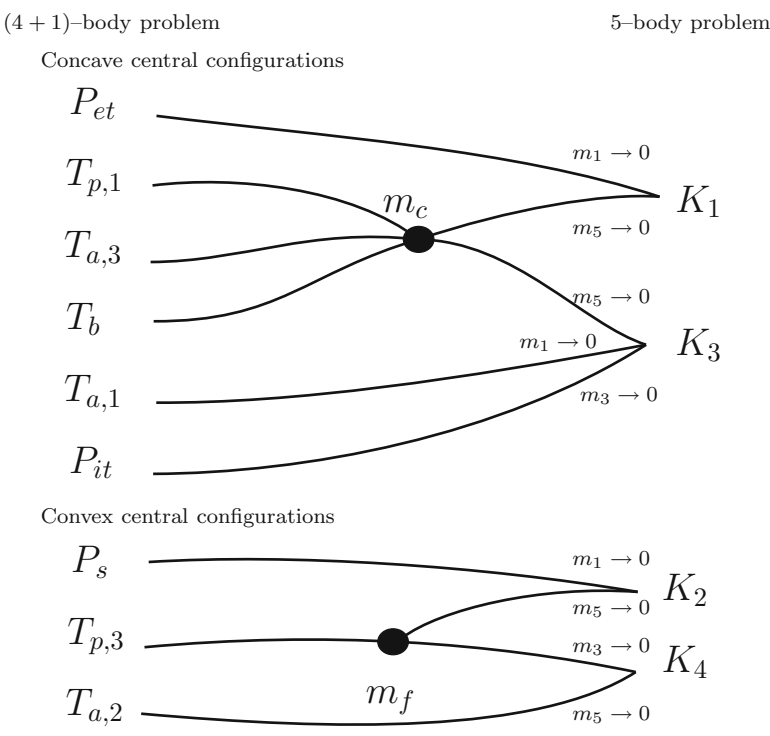

(b) From the degenerate central configuration with $m=m_{c}$ bifurcate 5 different (up to rotations and scalings) families of central configurations (see Fig. 7).

(c) From the degenerate central configuration with $m=m_{f}$ bifurcate 3 different (up to rotations and scalings) families of central configurations (see Fig. 7).

The bifurcation at $m=m_{c}$ has been first studied by Schmidt (1988). He found four families of central configurations bifurcating from the regular tetrahedron with one mass at its barycenter, each one symmetric with respect to one of the four axis of symmetry of the regular tetrahedron. Later on Santos (2004) completes the study of Schmidt (1988). In particular, after a complete analysis of the symmetries of the problem and using the equivariant branching lemma, he found seven symmetric families of central configurations bifurcating from the regular tetrahedron with one mass at its barycenter, four presenting a so-called axis-type symmetry (they are symmetric with respect to the straight line passing through a vertex and the barycenter of the tetrahedron) which correspond to the four families found by Schmidt (1988); and three presenting a so-called planar-type symmetry (they have two planes of symmetry and the intersection of these two planes is a straight line passing through the middle points of two opposite edges of the tetrahedron formed by the four equal masses).

Remarks (1) The branch connecting the configurations $T_{b}$ with $K_{1}$ through the bifurcation $m_{c}$ corresponds to the central configuration formed by the vertices of a regular tetrahedron and its barycenter.

(2) All the central configurations of the branch connecting the configurations $T_{a, 3}$ with $K_{3}$ through the bifurcation $m_{c}$ have the same symmetry satisfying $r_{12}=r_{13}=r_{14}, r_{23}=$ $r_{24}=r_{34}$ and $r_{25}=r_{35}=r_{45}$, which corresponds to a symmetry with respect to an axis passing through a vertex and the barycenter of the tetrahedron. This branch correspond to the one found by Schmidt (1988) given by the solution $a_{25}=a_{35}=a_{45}=\alpha$ of page 72. This branch corresponds also to one of the four axis-type symmetric branches given by Theorem 4.22 in Santos (2004), the one with $\Delta_{2}=\Delta_{3}=\Delta_{4}$. The other three 
solutions of Schmidt (1988), $a_{25}=-a_{35} / 3=a_{45}=\alpha,-a_{25} / 3=a_{35}=a_{45}=\alpha$ and $a_{25}=a_{35}=-a_{45} / 3=\alpha$ correspond to the other three axis-type symmetric branches in Santos (2004), namely the branches with $\Delta_{1}=\Delta_{2}=\Delta_{4}, \Delta_{1}=\Delta_{3}=\Delta_{4}$ and $\Delta_{1}=\Delta_{2}=\Delta_{3}$ respectively. The four branches are the same after renaming conveniently the masses. So they are represented only once in Fig. 7.

(3) All the central configurations of the branch connecting the configurations $T_{p, 1}$ with the bifurcation $m_{c}$ have the same symmetry satisfying $r_{13}=r_{14}=r_{23}=r_{24}, r_{35}=r_{45}$ and $r_{15}=r_{25}$, which corresponds to a symmetry with respect to a straight line passing through the middle points of two opposite edges of the tetrahedron formed by the four equal masses. This branch corresponds to one of the three families given by Theorem 4.22 in Santos (2004) presenting a planar-type symmetry. More precisely, it corresponds to the branch in Santos (2004) with $\Delta_{1}=\Delta_{2}$ and $\Delta_{3}=\Delta_{4}$. The other two branches (the one with $\Delta_{1}=\Delta_{3}$ and $\Delta_{2}=\Delta_{4}$ and the one with $\Delta_{1}=\Delta_{4}$ and $\Delta_{2}=\Delta_{3}$ ) are the same after renaming conveniently the masses. In Fig. 7 we only present one of these branches.

We conjecture that all the symmetric classes of central configurations of the 5-body problem with four equal masses to one and the non-equal mass varying in $[0,1]$ are represented in Fig. 7. If there are any other additional symmetric classes, these should neither start nor end in the central configurations of the spatial 5-body problem with equal masses or in the ones of the spatial $(4+1)$-body problem with four equal masses and one infinitesimal. They must start or or end in either the bifurcations $m_{c}, m_{f}$ or in some bifurcation value $m=m^{*} \neq m_{c}, m_{f}$.

In fact, analyzing the degenerate configuration with $m=m_{f}$ we see that there are no other classes of central configurations bifurcating from $m=m_{f}$. Indeed, the configuration $\left(q_{f}, m_{f}\right)$ corresponds to a simple branch point (see for instance Section 4.1 in Beyn et al. $2001)$; that is, there are exactly two distinct solution branches passing through $\left(q_{f}, m_{f}\right)$, the branch connecting $T_{p, 3}$ with $K_{4}$ and an additional branch connecting two different representatives of the class $K_{2}$.

\section{Continuation from the (5)-body problem to the $(1+4)$-body problem}

The restricted $(1+4)$-body problem is a limiting case of the 5-body problem having one unit mass and four infinitesimal masses. Albouy and Llibre (2002) studied the restricted $(1+4)$ body problem when the four infinitesimal masses are equal. They found the five classes of central configurations detailed below.

$R_{1}$ : $(2 \mathrm{cc})$ The four infinitesimal masses are at the vertices of a regular tetrahedron and the larger mass is at its barycenter.

$R_{2}$ : $(6 \mathrm{cc})$ The masses are at the vertices of a regular pyramid with a square base, the infinitesimal masses are at the vertices of the square.

$R_{3}$ : $(8 \mathrm{cc}$ ) The four infinitesimal masses are at the vertices of a regular pyramid with an equilateral triangle base, the larger mass lies in the interior of the pyramid and on its axis of symmetry.

$R_{4}$ : $(8 \mathrm{cc}$ ) Three infinitesimal masses form an equilateral triangle. The other two masses are on the axis orthogonal to the plane defined by the triangle passing through its barycenter, one above the plane and the other one below. 
$R_{5}$ : (24 cc) Three infinitesimal masses form an isosceles triangle. The other two masses are on the axis orthogonal to the plane defined by the triangle passing through the barycenter of the triangle, one above the plane and the other one below.

In Alvarez-Ramírez et al. (2008) the authors continue numerically the symmetric central configurations of the spatial 5-body problem to the restricted spatial $(1+4)$-body problem with four infinitesimal masses. Here we have repeated the computations and the results made in Alvarez-Ramírez et al. (2008) and we have corrected some mistakes. Thus here we present the improved results of Alvarez-Ramírez et al. (2008), which are detailed below and they are represented in Fig. 8. We also have analyzed the symmetries of the continued families.

$K_{1}$ : When we fix $m_{5}=1$, the representative of the class $K_{1}$ can be continued to a family that ends at a representative of the class $R_{1}$. All the configurations of this family consists of a regular tetrahedron with four masses equal to 1 and the variable mass at its barycenter. When we fix $m_{2}=1$, the representative of the class $K_{1}$ can be continued to a family that ends at a degenerate configuration $q_{a}$ (see Fig. 9) with $m=m_{a}=0.94120559 \ldots$ and with mutual distances

$$
\begin{array}{lll}
r_{12}=r_{23}=r_{24}=1.69174590 \ldots, & & r_{13}=r_{14}=r_{34}=1.59766522 \ldots, \\
r_{15}=r_{35}=r_{45}=1.025631876 \ldots, & r_{25}=0.969738526 \ldots
\end{array}
$$

All the configurations of this family satisfy the symmetry $r_{13}=r_{14}=r_{34}, r_{15}=$ $r_{35}=r_{45}, r_{12}=r_{23}=r_{24}$.

Fig. 8 Connections between the classes of central configurations of the (5)-body problem with equal masses and the ones of the spatial $(1+4)$-body problem with equal infinitesimal masses. Each line corresponds to a different family of central configurations of the 5-body problem with 4 equal masses. The black dots represent mass values where degenerate central configurations occur

Fig. 9 The degenerate central configuration $q_{a}$

Concave central configurations
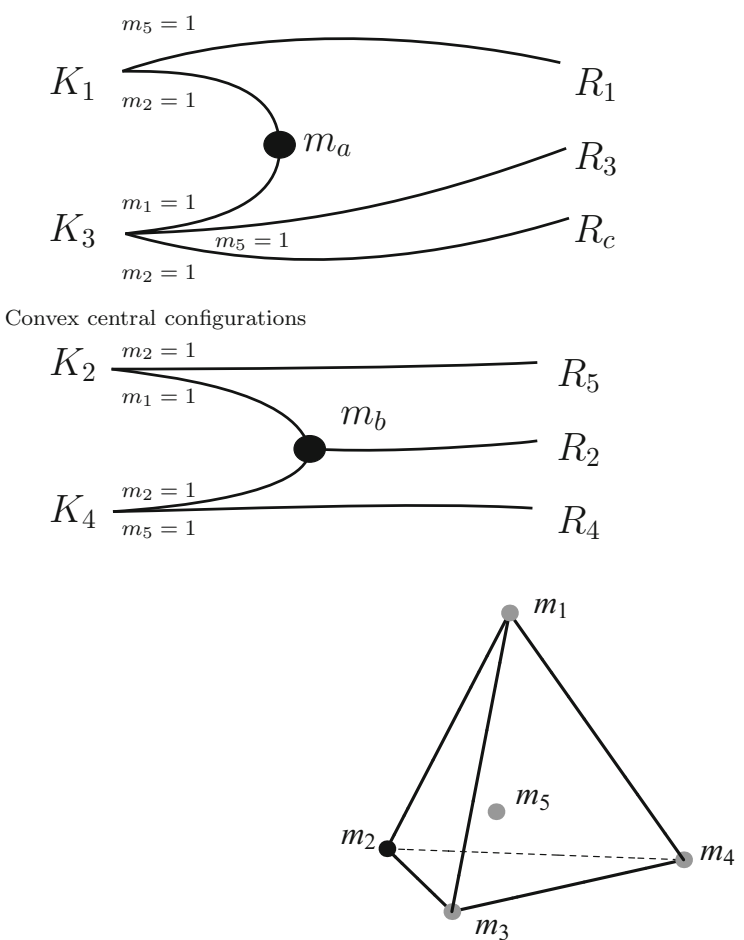
$K_{2}$ : When we fix $m_{2}=1$, the representative of the class $K_{2}$ can be continued to a family that ends at a representative of the class $R_{5}$. All the configurations of this family satisfy the symmetry $r_{13}=r_{15}, r_{23}=r_{25}, r_{34}=r_{45}$.

When we fix $m_{1}=1$, the representative of the class $K_{2}$ can be continued to a family that ends at a representative of the class $R_{2}$ by passing trough a degenerate configuration $q_{b}$ with $m=m_{b}=0.59623579 \ldots$. All the configurations of this family consists of a pyramid with square base, with the four equal masses on the square. The mutual distances corresponding to the configuration with $m=m_{b}$ are

$$
\begin{aligned}
& r_{12}=r_{13}=r_{14}=r_{15}=1.81043914 \ldots, \\
& r_{23}=r_{25}=r_{34}=r_{45}=1.58951902 \ldots
\end{aligned} \quad r_{24}=r_{35}=2.24791936 \ldots,
$$

$K_{3}$ : When we fix $m_{1}=1$, the representative of the class $K_{3}$ can be continued to a family that ends at a representative of the degenerate central configuration with $m=m_{a}$. All the configurations of this family satisfy the symmetry $r_{13}=r_{14}, r_{23}=r_{24}, r_{35}=r_{45}$. When we fix $m_{5}=1$, the representative of the class $K_{3}$ can be continued to a family that ends at a representative of the class $R_{3}$. All the configurations of this family satisfies the symmetry $r_{12}=r_{13}=r_{14}, r_{23}=r_{24}=r_{34}, r_{25}=r_{35}=r_{45}$.

When we fix $m_{2}=1$, the representative of the class $K_{3}$ can be continued to a family that ends at a coalescent central configuration with the larger mass and two infinitesimal masses located at three different vertices of a tetrahedron and two infinitesimal masses coalescing at the remaining vertex the tetrahedron. This class of coalescent central configurations is denoted by $R_{c}$. Notice that the configurations in $R_{c}$ are in the boundary between convex and nonconvex.

$K_{4}$ : When we fix $m_{2}=1$, the representative of the class $K_{4}$ can be continued to a family that ends at a representative of the degenerate central configuration with $m=m_{b}$. All the configurations of this family satisfy the symmetry $r_{12}=r_{25}, r_{13}=r_{14}, r_{23}=r_{24}$, $r_{35}=r_{45}$.

When we fix $m_{5}=1$, the representative of the class $K_{4}$ can be continued to a family that ends at a representative of the class $R_{4}$. All the configurations of this family satisfy the symmetry $r_{12}=r_{13}=r_{14}, r_{23}=r_{24}=r_{34}, r_{25}=r_{35}=r_{45}$.

Notice that all this families of central configurations are symmetric.

Analyzing the degenerate configurations $q_{a}$ and $q_{b}$, we have that the configuration $q_{a}$ with $m=m_{a}$ is a simple fold (see Section 3.2 in Beyn et al. 2001); that is, there is a unique solution branch passing through $\left(q_{a}, m_{a}\right)$ the branch connecting a representative of the class $K_{1}$ with a representative of the class $K_{3}$. The configuration $q_{b}$ with $m=m_{b}$ is a simple branch point (see again Section 4.1 in Beyn et al. 2001); that is, there are exactly two distinct solution branches passing through $\left(q_{b}, m_{b}\right)$, the branch connecting a representative of the class $K_{2}$ with a representative of the class $R_{2}$ and an additional branch connecting two different representatives of the class $K_{4}$.

We conjecture that all the symmetric classes of central configurations of the 5-body problem with four masses equal to $m$ and the non-equal mass equal to 1 when $m$ varies in $[1,0]$ are represented in Fig. 8. If there are any other additional symmetric classes, these should neither start nor end in the central configurations of the spatial 5-body problem with equal masses or in the ones of the spatial $(1+4)$-body problem with four infinitesimal equal masses.

Acknowledgments The first author is supported by the project grant Red de cuerpos académicos Ecuaciones Diferenciales. Proyecto sistemas dinámicos y estabilización. PROMEP 2011-SEP, Mexico. The second author is partially supported by MINECO Grant Number MTM2013-40998-P. The third author is partially supported by MINECO grant number MTM2013-40998-P, by AGAUR Grant Number 2014SGR 568, two FP7-PEOPLE- 
2012-IRSES Numbers 316338 and 318999, and MINECO/FEDER Grant Number UNAB10-4E-378. We thank to the anonymous reviewer for his/her valuable comments and suggestions.

\section{References}

Albouy, A.: Symétrie des configurations centrales de quatre corps. C. R. Acad. Sci. Paris Sér. I Math. 320, 217-220 (1995)

Albouy, A.: Reserches sur le probléme des n corps. Notes scientifiques et techniques du Bureau des Longitudes, Paris (1997)

Albouy, A.: On a paper of Moeckel on central configurations. Regul. Chaotic Dyn. 8, 133-142 (2003)

Albouy, A., Fu, Y.: Euler configurations and quasi polynomial systems. Regul. Chaotic Dyn. 12, 39-55 (2007)

Albouy, A., Llibre J.: Spatial central configurations for the $(1+4)$-body problem. In: Celestial Mechanics (Evanston, IL, 1999), pp. 1-16. Contemp. Math., vol. 292, Amer. Math. Soc., Providence, RI (2002)

Alvarez-Ramírez, M., Delgado, J., Llibre, J.: On the spatial central configurations of the 5-body problem and their bifurcations. Discrete Contin. Dyn. Syst. Ser. S 1, 505-518 (2008)

Bang, D., Elmabsout, B.: Representations of complex functions, means on the regular n-gon and applications to gravitational potential. J. Phys. A 36, 11435-11450 (2003)

Beyn, W.-J., Champneys, A., Doedel, E.J., Kuznetsov, Y.A., Sandstede, B., Govaerts, W.: Numerical continuation and computation of normal forms. In: Fiedler, B. (ed.) Handbook of Dynamical Systems III: Towards Applications. Elsevier, Amsterdam (2001). Chap. 4

Chenciner, A.: Collisions totales, mouvements complètement paraboliques et réduction des homothéthies dans le problème des $n$ corps. Regul. Chaotic Dyn. 3, 93-106 (1998)

Faugère, J.C., Kotsireas, I.: Symmetry Theorems for the Newtonian 4- and 5-Body Problems with Equal Masses. Computer Algebra in Scientific Computing CASC'99 (Munich). Springer, Berlin (1999)

Fayçal, N.: On the classification of pyramidal central configurations. Proc. Am. Math. Soc. 124, 249-258 (1996)

Hampton, M., Santoprete, M.: Seven-body central configurations: a family of central configurations in the spatial seven-body problem. Celestial Mech. Dyn. Astron. 99, 293-305 (2007)

Kotsireas, I., Lazard, D.: Central configurations of the 5-body problem with equal masses in three-dimensional space. J. Math. Sci. 108, 1119-1138 (2002)

Leandro, E.: On the Dziobek configurations of the restricted $(N+1)$-body problem with equal masses. Discrete Contin. Dyn. Syst. Ser. S 1, 589-595 (2008)

Lee, T.L., Santoprete, M.: Central configurations of the five-body problem with equal masses. Celestial Mech. Dyn. Astron. 104, 369-381 (2009)

Lehmann-Filhés, R.: Ueber zwei Fälle des Vielkörperproblems. Astron. Nachr. 127, 137-143 (1891)

Llibre, J.: Posiciones de equilibrio relativo del problema de 4 cuerpos. Publicacions Matemàtiques 3, 73-88 (1976)

Saari, D., Hulkower, N.D.: On the manifolds of total collapse orbits and of completely parabolic orbits for the n-body problem. J. Diff. Equ. 41, 27-43 (1981)

Santos, A.A.: Dziobek's configurations in restricted problems and bifurcation. Celest. Mech. Dyn. Astron. 90, 213-238 (2004)

Santos, A.A., Vidal, C.: Symmetry of the restricted $(4+1)$-body problem with equal masses. Regul. Chaotic Dyn. 12, 27-38 (2007)

Schmidt, D.S.: Central configurations in $\mathbb{R}^{2}$ and $\mathbb{R}^{3}$. In: Hamiltonian Dynamical Systems (Boulder, CO, 1987), pp. 59-76. In Contemp. Math., 81, Amer. Math. Soc., Providence, RI (1988)

Tsai, Y.: Dziobek configurations of the restricted $(N+1)$-body problem with equal masses. J. Math. Phys. 53, 072902 (2012) 